\title{
On fine differentiability properties of horizons and applications to Riemannian geometry
}

\author{
Piotr T. Chruściel ${ }^{\text {a, },}$, Joseph H.G. Fu ${ }^{\text {b,2, }}$, \\ Gregory J. Galloway ${ }^{\mathrm{c}, *, 3}$, Ralph Howard ${ }^{\mathrm{d}, 4}$ \\ a Département de Mathématiques, Faculté des Sciences, Parc de Grandmont, F37200 Tours, France \\ ${ }^{\mathrm{b}}$ Department of Mathematics, University of Georgia, Athens, GA 30602, USA \\ ${ }^{c}$ Department of Mathematics, University of Miami, Coral Gables, FL 33124, USA \\ ${ }^{d}$ Department of Mathematics, University of South Carolina, Columbia, SC 29208, USA
}

Received 1 December 2000

\begin{abstract}
We study fine differentiability properties of horizons. We show that the set of end points of generators of an $n$-dimensional horizon $\mathcal{H}$ (which is included in an $(n+1)$-dimensional space-time $M$ ) has vanishing $n$-dimensional Hausdorff measure. This is proved by showing that the set of end points of generators at which the horizon is differentiable has the same property. For $1 \leq k \leq n+1$, we show (using deep results of Alberti) that the set of points where the convex hull of the set of generators leaving the horizon has dimension $k$ is "almost a $C^{2}$ manifold of dimension $n+1-k$ ": it can be covered, up to a set of vanishing $(n+1-k)$-dimensional Hausdorff measure, by a countable number of $C^{2}$ manifolds. We use our Lorentzian geometry results to derive information about the fine differentiability properties of the distance function and the structure of cut loci in Riemannian geometry. () 2002 Elsevier Science B.V. All rights reserved.
\end{abstract}

Keywords: Riemannian geometry; Horizons; Hausdorff measure

\section{Introduction}

Horizons are amongst the most important objects that one encounters in causality theory: Cauchy horizons constitute boundaries beyond which predictability breaks down; event

\footnotetext{
* Corresponding author.

E-mail addresses: chrusciel@univ-tours.fr (P.T. Chruściel), fu@ math.uga.edu (J.H.G. Fu), galloway@math.miami.edu (G.J. Galloway), howard@math.sc.edu (R. Howard).

${ }^{1}$ Supported in part by KBN grant No. 2 P03B 13016.

${ }^{2}$ Supported in part by NSF grant No. DMS-9972094.

${ }^{3}$ Supported in part by NSF grant No. DMS-9803566.

${ }^{4}$ Supported in part by DoD grant No. N00014-97-1-0806.
} 
horizons are boundaries beyond which no return is possible. The key structural property of horizons is the existence of generators: recall that an embedded hypersurface $\mathcal{H} \subset M$ is said to be future null geodesically ruled if every point $p \in \mathcal{H}$ belongs to a future inextensible null geodesic $\Gamma \subset \mathcal{H}$; those geodesics are called generators of $\mathcal{H}$. One can then extract the essential properties of Cauchy horizons, or black hole event horizons, in the following definition: $\mathcal{H}$ is a future horizon if $\mathcal{H}$ is an achronal, closed, future null geodesically ruled topological hypersurface. It follows from the above definition (or from the properties of past Cauchy horizons, or from the properties of future event horizons) that the generators can have past endpoints on $\mathcal{H}$, but no future endpoints.

The set $\mathcal{H}_{\text {end }}$ of end points of generators of $\mathcal{H}$ provides an important tool in the study of the structure of horizons; for simplicity, we will refer to those points as end points. In particular, one wants to know how "large" this set can be. One defines the multiplicity $N(p)$ of a point $p \in \mathcal{H}$ as the number of generators which pass through or exit $\mathcal{H}$ at $p$; it is well known that if $N(p)>1$, then $p$ is necessarily the endpoint of all relevant generators. The set of points with multiplicity $N(p)>1$ determines the differentiability properties of horizons: as has been shown by Beem and Królak [2], horizons are non-differentiable precisely at this set. It is also well known that the set of points at which a horizon is non-differentiable has vanishing $n$-dimensional Hausdorff measure, and this gives one control over the size of the set of endpoints with multiplicity $N(p)>1$. Thus, in order to control the dimension of $\mathcal{H}_{\mathrm{end}}$ it remains to estimate that of the set of endpoints with multiplicity $N(p)=1$. Let us denote by $\mathcal{H}_{\text {diff }}$ the set of points of $\mathcal{H}$ at which $\mathcal{H}$ is differentiable; what has been said shows that the set of endpoints with multiplicity $N(p)=1$ coincides with the set $\mathcal{H}_{\text {end }} \cap \mathcal{H}_{\text {diff }}$. Beem and Królak [2] have displayed an example of a horizon with an end point with multiplicity 1 (at which $\mathcal{H}$ is, of course, differentiable), thus there exist horizons for which the set $\mathcal{H}_{\text {end }} \cap \mathcal{H}_{\text {diff }}$ is not empty. Our first main result is the following theorem.

Theorem 1. Let $\mathcal{H}$ be a future horizon in an $(n+1)$-dimensional space-time $(M, g)$. Then the set $\mathcal{H}_{\text {end }} \cap \mathcal{H}_{\text {diff }}$ has vanishing $n$-dimensional Hausdorff measure. Moreover, for any $C^{2}$ space-like hypersurface $\mathcal{S}$ the set $\mathcal{H}_{\mathrm{end}} \cap \mathcal{H}_{\mathrm{diff}} \cap \mathcal{S}$ has vanishing $(n-1)$-dimensional Hausdorff measure.

Remark 2. In fact, we expect a stronger conclusion to hold, namely that the set $\mathcal{H}_{\text {end }} \cap \mathcal{H}_{\text {diff }}$ has vanishing $(n-1)$-dimensional Hausdorff measure in $\mathcal{H}$. The rationale for this is as follows: we first note that it is possible to weaken the regularity assumption on $\mathcal{S}$. For Theorem 1 to hold it is sufficient to require that $\mathcal{S}$ be a Lipschitz hypersurface that meets the horizon transversely at almost all points of the intersection. This point, taken in conjunction with the fact that the set $\mathcal{H}_{\text {end }} \cap \mathcal{H}_{\text {diff }}$ is necessarily acausal, suggests the possibility of constructing such a Lipschitz hypersurface (or perhaps a countable collection of such Lipschitz hypersurfaces) that contains $\mathcal{H}_{\text {end }} \cap \mathcal{H}_{\text {diff }}$, at least up to a set of vanishing $(n-1)$-dimensional Hausdorff measure. Theorem 1, under this weaker regularity assumption on $\mathcal{S}$ would then give the strengthened conclusion. Initial efforts to construct such a hypersurface have not been successful.

The set of points where the multiplicity $N(p)$ is large has a more precise structure. To describe this, equip $M$ with an auxiliary complete Riemannian metric $\sigma$, and for each 
$p \in \mathcal{H}$, let $\mathcal{N}_{p}^{+}$be the set of future pointing $\sigma$-unit vectors that are tangent to a generator of $\mathcal{H}$ at $p$. We call such vectors semi-tangents to $\mathcal{H}$. Then the number of vectors in $\mathcal{N}_{p}^{+}$is just the multiplicity $N(p)$ of $p$. Define

$$
\mathcal{C}_{p}:=\text { convex cone generated by } \mathcal{N}_{p}^{+} .
$$

We can measure the size of the set of generators through $p$ by $\operatorname{dim}\left(\mathcal{C}_{p}\right)$ (which is the dimension of the linear span of $\mathcal{C}_{p}$ in $\left.T_{p} M\right)$. This is a different measure than $N(p)$; in particular, this gives finer information when $N(p)=\infty$. We also set

$$
\mathcal{H}[k]:=\left\{p: \operatorname{dim}\left(\mathcal{C}_{p}\right) \geq k\right\} .
$$

For $k=1, \mathcal{H}[k]=\mathcal{H}$ as every point is on at least one generator. For $k=2, \mathcal{H}[k]$ is the set of points of $\mathcal{H}$ that are on more than one generator. As $\operatorname{dim}\left(\mathcal{C}_{p}\right)$ is the dimension of the span of $\mathcal{N}_{p}^{+}$and $\mathcal{N}_{p}^{+}$contains $N(p)$ vectors, $\operatorname{dim}\left(\mathcal{C}_{p}\right) \leq N(p)$. This implies

$$
\mathcal{H}[k] \subseteq\{p \in \mathcal{H}: N(p) \geq k\}
$$

Also, for $1 \leq k \leq 3$, any $k$ distinct elements of $\mathcal{N}_{p}^{+}$are linearly independent. ${ }^{5}$ Therefore if $1 \leq k \leq 3$ and $N(p) \geq k$ then choosing $k$ distinct, and thus linearly independent, elements of $\mathcal{N}_{p}^{+}$, shows that $\operatorname{dim}\left(\mathcal{C}_{p}\right) \geq k$. Whence

$$
\mathcal{H}[k]=\{p \in \mathcal{H}: N(p) \geq k\} \text { for } 1 \leq k \leq 3 .
$$

Our next main result, based on the deep results ${ }^{6}$ in [1], is that the sets $\mathcal{H}[k]$ are "almost $C^{2}$ submanifolds of dimension $n+1-k$, up to singular sets of lower dimension". 7 To make this statement precise, let $\mathfrak{h}^{m}$ be the $m$-dimensional Hausdorff measure on $M$ (defined with respect to some Riemannian metric $\sigma$ on $M$ ). Recall [1, Definition 1.1, p. 19] a Borel set $\Sigma \subset M$ is a $\left(\mathfrak{h}^{m}, m\right)$ rectifiable set of class $C^{2}$ iff $\Sigma$ can be covered, up to a set of vanishing $\mathfrak{h}^{m}$ measure, by a countable collection of $m$-dimensional $C^{2}$ submanifolds of $M$. This definition is independent of the choice of the Riemannian metric $\sigma$. Following [1], we will shorten " $\left(\mathfrak{h}^{m}, m\right)$ rectifiable set of class $C^{2}$ " to " $C^{2}$ rectifiable of dimension $m$ ".

Theorem 3. For $1 \leq k \leq n+1$ the set $\mathcal{H}[k]$ is a $C^{2}$ rectifiable set of dimension $n+1-k$. Therefore $\mathcal{H}[k]$ has Hausdorff dimension $\leq n+1-k$.

Using $k=1$, and that $\mathcal{H}[1]=\mathcal{H}$, this implies that horizons are $C^{2}$ rectifiable of dimension $n$. As they are also locally Lipschitz graphs they have the further property that $\mathfrak{h}^{n}(\mathcal{H} \cap K)<$ $\infty$ for all compact sets $K \subseteq M$. When $k=n+1$ this implies that $\mathcal{H}[n+1]$ is a countable set (cf. Remark 7).

\footnotetext{
${ }^{5}$ The linear span of two future pointing $\sigma$-unit null vectors is a two-dimensional time-like subspace and there are only two future pointing null rays in this subspace. So a third future pointing $\sigma$-unit vector cannot be in the span of the first two.

${ }^{6}$ The reader is warned that the dimension index $k$, in $\mathcal{H}[k]$, is shifted by one, as compared to that used in Theorem 3 of Alberti [1]; compare the remarks following Definitions 1.5 and 1.7 in [1].

${ }^{7}$ We note a related result of Husa and Winicour [12], where it is shown that the set $\{N(p)=2\}$ is, up to a lower dimensional set, a smooth submanifold of co-dimension two for the horizons considered there.
} 


\section{Proof of Theorem 1}

We shall prove the second part of the theorem; the first part follows immediately from the second and the co-area formula. Let, then, $\mathcal{S}$ be as in Theorem 1; since the result is purely local, without loss of generality we may assume that $\mathcal{S}$ is the level set $\{t=1\}$ of a time function $t$, with range $\mathbb{R}$, the level sets of which are Cauchy surfaces. We use the constructions and notations of Chruściel et al. [4], with $\Sigma_{1}=\mathcal{S}$ and $\Sigma_{2}=\{t=2\}$. Let

$$
\hat{S}_{1}=\mathcal{S} \cap \mathcal{H}_{\text {end }} \cap \mathcal{H}_{\text {diff }},
$$

and let $A, \phi$ be defined as at the beginning of the proof of Theorem 6.1 in [4]. Hence, $A$ is the subset of $S_{2}=\Sigma_{2} \cap \mathcal{H}$ consisting of those points in $S_{2}$ that are met by the generators of $\mathcal{H}$ that meet $S_{1}=\Sigma_{1} \cap \mathcal{H}$, and $\phi: A \rightarrow S_{1}$ is the map that moves the points of $A$ back along these generators to $S_{1}$. We can choose the auxiliary Riemannian metric $\sigma$ on $M$ so that $\mathrm{d} t$ has unit length with respect to this metric. Then $A$ is an $A_{\delta}$ set as defined by Eq. (6.6) of Chruściel et al. [4], with $\delta=1$. We set

$$
\hat{A}=\phi^{-1}\left(\hat{S}_{1}\right) \subset A \subset S_{2},
$$

thus, the points in $\hat{A}$ are precisely those points on $\Sigma_{2} \cap \mathcal{H}$ the generators through which exit $\mathcal{H}$, when followed to the past, at the differentiable end points on $\mathcal{S}$.

For $i=1,2$, let $\mathfrak{h}_{h_{i}}^{n-1}$ denote the $(n-1)$-dimensional Hausdorff measure on $\Sigma_{i}$ with respect to the distance function determined by the induced metric $h_{i}$ on $\Sigma_{i}$. By a straightforward extension of the proof of Chruściel et al. [4, Proposition 6.14], one has for any $\mathfrak{h}_{h_{2}}^{n-1}$-measurable subset $\Omega$ of $A$ :

$$
\int_{S_{1}} N\left(p, S_{2}\right) \mathbf{1}_{\phi(\Omega)} \mathrm{dh}_{h_{1}}^{n-1}(p)=\int_{\Omega} J(\phi)(q) \mathrm{dh}_{h_{2}}^{n-1}(q),
$$

where $\mathbf{1}_{U}$ denotes the characteristic function of the set $U$, and $J(\phi)$ is, in a suitably defined sense (cf. [4, Proposition 6.14]), the Jacobian of the locally Lipschitz function $\phi$.

In Proposition 4, we show that there exists a $\mathfrak{h}_{h_{2}}^{n-1}$-negligible set $\hat{A}^{\prime} \subset \hat{A}$ such that $J(\phi)(q)=0$ for all $q \in \hat{A} \backslash \hat{A}^{\prime}$. It then follows that:

$$
\hat{A} \subset \Omega \equiv\{q \in A: J(\phi)=0\} \cup \hat{A}^{\prime},
$$

$\Omega$, as defined above, is the union of a $\mathfrak{h}_{h_{2}}^{n-1}$-measurable set and a $\mathfrak{h}_{h_{2}}^{n-1}$-negligible set, and hence is itself $\mathfrak{h}_{h_{2}}^{n-1}$-measurable. Eq. (2.1) then shows that $\phi(\Omega)$ is $\mathfrak{h}_{h_{1}}^{n-1}$-negligible. Now, since $\hat{S}_{1} \subset \phi(\Omega)$, the result follows.

It thus remains to establish the following proposition.

Proposition 4. $J(\phi)(q)=0$ for $\mathfrak{h}_{h_{2}}^{n-1}$-almost all $q \in \hat{A}$.

Proof. We use the definitions, constructions and notations of the proof of Chruściel et al. [4, Proposition 6.16]. Thus, let $\mathcal{U} \subset \Sigma_{2}$ be a coordinate neighborhood of the form $\mathcal{V} \times(a, b)$ with $\mathcal{V} \subset \mathbb{R}^{n-1}$ and $a, b \in \mathbb{R}$, in which $\mathcal{U} \cap N$ is the graph of a $C^{1,1}$ function $g: \mathcal{V} \rightarrow \mathbb{R}$, and in which $\mathcal{H} \cap \mathcal{U}$ is the graph of a semi-convex function $f: \mathcal{V} \rightarrow \mathbb{R}$. Here, $N=N_{\delta}$ 
is a locally $C^{1,1}$ hypersurface in $\Sigma_{2}$ into which $A$ has been embedded. Let pr $A$ denote the projection onto $\mathcal{V}$ of $A \cap \mathcal{U}$, thus $A \cap \mathcal{U}$ is the graph of $g$ over $\operatorname{pr} A$.

Now, let $x_{0} \in \hat{B} \cap \operatorname{pr} \hat{A}$, where $\hat{B}$ is the set of full measure in pr $A$ constructed in the proof of Chruściel et al. [4, Proposition 6.16], and $\operatorname{pr} \hat{A}$ is the projection onto $\mathcal{V}$ of $\hat{A} \cap \mathcal{U}$. Since $g$ is Lipschitz, the graph of $g$ over $\hat{B} \cap \operatorname{pr} \hat{A}$ has full measure in $\hat{A}$. Let $q_{0}=\left(x_{0}, f\left(x_{0}\right)\right) \in \hat{A}$ be the corresponding point on $\mathcal{H} \cap \Sigma_{2}$, thus the generator $\Gamma$ of $\mathcal{H}$ passing through $q_{0}$ exits the horizon at a point $p \in \mathcal{S} \cap \mathcal{H}_{\text {diff }}$. Let $\hat{\Gamma}$ be any null geodesic which extends $\Gamma$ to the past, and let $p_{n}$ be any sequence of points on $\hat{\Gamma}$ which are to the causal past of $p$ and which approach $p$ as $n$ tends to infinity. Since $\hat{\Gamma}$ is a null geodesic which exits $\mathcal{H}$ at $p$, the $p_{n}$ 's lie to the time-like past $I^{-}(\mathcal{H})$ of $\mathcal{H}$. Thus the integral curve $\gamma_{n}$ of $\partial / \partial t$ starting at $p_{n}$ meets $\mathcal{H}$ at some point $r_{n} \in \gamma_{n} \cap \mathcal{H}$. One can then construct a causal curve from $p_{n}$ to $\mathcal{H} \cap \Sigma_{2}$ by following $\gamma_{n}$ from $p_{n}$ to $r_{n}$, and any generator of $\mathcal{H}$ passing through $r_{n}$; as $(M, g)$ is globally hyperbolic this generator will necessarily intersect $\Sigma_{2}$. It follows that there exists a time-like curve $\hat{\gamma}_{n} \subset J^{-}(\mathcal{H})$ from $p_{n}$ to $\mathcal{H} \cap \Sigma_{2}$. By the compactness of the space of causal curves, passing to a subsequence if necessary, the $\hat{\gamma}_{n}$ 's converge (in a well-known sense) to a causal curve $\gamma$ from $p$ to a point $q \in \mathcal{H} \cap \Sigma_{2}$. The achronality of $\mathcal{H}$ shows that $\gamma$ is a generator of $\mathcal{H}$ passing through $p$, hence $\gamma=\Gamma$ and $q=q_{0}$.

Now suppose that $J(\phi)\left(q_{0}\right) \neq 0$. By the construction of the set $\hat{B}$, there exists $g_{j} \in$ $C^{2}(\mathcal{V})$, approximating $g$, such that $S_{j}$, the graph of $g_{j}$, is a $C^{2}$ hypersurface in $\Sigma_{2}$ which, in a well-defined sense, makes second-order contact with $\mathcal{H} \cap \Sigma_{2}$ at $q_{0}$. (More precisely, $g_{j}$ and $f$, as well as their first derivatives, agree at $q_{0}$, and the second derivative of $g_{j}$ agrees with the so-called second Alexandrov derivative of $f$ at $q_{0}$.) Since $S_{j}$ is tangent to $\mathcal{H} \cap \Sigma_{2}$ at $q_{0}$, the null geodesic $\hat{\Gamma}$ is normal to $S_{j}$ at $q_{0}$. Let $\phi_{j}: S_{j} \rightarrow \mathcal{S}$ be the $C^{1}$ map which moves the points of $S_{j}$ along the family of null geodesics normal to $S_{j}$ which includes $\hat{\Gamma}$. Then we have $J\left(\phi_{j}\left(q_{0}\right)\right)=J(\phi)\left(q_{0}\right) \neq 0$, cf. [4, Eq. (6.40)]. Define $g_{j, \varepsilon} \in C^{2}(\mathcal{V})$ by

$$
g_{j, \varepsilon}(x)=g_{j}(x)+\varepsilon\left|x-x_{0}\right|^{2},
$$

and let $S_{j, \varepsilon}$ be the graph of $g_{j, \varepsilon}$; for $\varepsilon>0, S_{j, \varepsilon} \subset J^{+}(\mathcal{H})$ and $S_{j, \varepsilon} \backslash\left\{q_{0}\right\} \subset I^{+}(\mathcal{H})$. Note that as $S_{j, \varepsilon}$ is tangent to $S_{j}$ at $q_{0}, \hat{\Gamma}$ is normal to $S_{j, \varepsilon}$ at $q_{0}$.

The fact that the Jacobian of $\phi_{j}$ is non-zero at $q_{0}$ implies that $p$ is not a focal point to $S_{j}$ along $\hat{\Gamma}$. Moreover, there can be no focal points to $S_{j}$ along the segment of $\hat{\Gamma}$ to the future of $p$, cf. [4, Lemma 4.15]. It follows that by taking $\varepsilon$ small enough and $\hat{\Gamma}$ short enough, there will be no focal points to $S_{j, \varepsilon}$ along $\hat{\Gamma}$. This implies by normal exponentiation that there exists an embedded $C^{2}$ null hypersurface $\mathcal{N}_{j, \varepsilon}$ which contains $\hat{\Gamma}$ and, by shrinking it if necessary, $S_{j, \varepsilon}$, as well, cf. [4, Proposition A3]. Moreover, there exists a neighborhood $\mathcal{O}$ of $\hat{\Gamma}$ in which $\mathcal{N}_{j, \varepsilon}$ is achronal: indeed, since space-time is time orientable, $\mathcal{N}_{j, \varepsilon}$ is a two-sided connected embedded hypersurface in $M$. As such $\mathcal{N}_{j, \varepsilon}$ admits a connected neighborhood $\mathcal{O}$ which is separated by $\mathcal{N}_{j, \varepsilon}\left(\mathcal{N}_{j, \varepsilon} \subset \mathcal{O}\right.$, and $\mathcal{O} \backslash \mathcal{N}_{j, \varepsilon}$ consists of two components). Then a future directed time-like curve joining points of $\mathcal{N}_{j, \varepsilon}$ would be a time-like curve from the future side of $\mathcal{N}_{j, \varepsilon}$ to the past side of $\mathcal{N}_{j, \varepsilon}$, which is impossible if the curve remains in $\mathcal{O}$. We conclude that $\mathcal{N}_{j, \varepsilon}$ is achronal in $\mathcal{O}$.

Consider now the time-like curves $\hat{\gamma}_{n} \subset J^{-}(\mathcal{H})$ constructed earlier in the proof; since the $\hat{\gamma}_{n}$ 's converge to $\Gamma$ there exists $n_{0}$ such that all the $\hat{\gamma}_{n}$ 's are entirely contained in $\mathcal{O}$ for $n \geq n_{0}$. Moreover, by taking $n_{0}$ larger if necessary, it is clear that each such $\hat{\gamma}_{n}$ will 
meet the hypersurface $P$ in $M$ obtained by pushing $S_{j, \varepsilon}$ to the past along the integral curves of $-(\partial / \partial t)$. One can then construct a time-like curve from $p_{n}$ to $S_{j, \varepsilon}$ contained in $\mathcal{O}$ by following $\hat{\gamma}_{n}$ from $p_{n}$ to $P$, and then an integral curve of $\partial / \partial t$ to $S_{j, \varepsilon}$. This contradicts the achronality of $\mathcal{N}_{j, \varepsilon}$ in $\mathcal{O}$, and establishes Proposition 4.

\section{Proof of Theorem 3}

We start by showing that $\mathcal{H}$ has no worse regularity than being the boundary of a convex set.

Proposition 5. For any point $p \in \mathcal{H}$ there is a coordinate system $x^{1}, \ldots, x^{n+1}$ defined on an open set $U \subseteq M$, so that $\mathcal{H} \cap U$ is given by the graph $x^{n+1}=h\left(x^{1}, \ldots, x^{n}\right)$ of a convex function $h$.

Proof. It is shown in [4, Theorem 2.2] that $\mathcal{H}$ is locally the graph of a semi-convex function. That is, there is a coordinate system $y^{1}, \ldots, y^{n+1}$, so that $U \cap \mathcal{H}$ is given by a graph $y^{n+1}=$ $u\left(y^{1}, \ldots, y^{n}\right)+h\left(x^{1}, \ldots, x^{n}\right)$, where $u$ is $C^{\infty}$ and $h$ is convex. Define new coordinates by $x^{i}=y^{i}$ for $i=1, \ldots, n$ and $x^{n+1}=y^{n+1}-u\left(x^{1}, \ldots, x^{n}\right)$. In these coordinates $\mathcal{H}$ is given by $x^{n+1}=h\left(x^{1}, \ldots, x^{n}\right)$.

Recall that a convex body in a finite dimensional vector space has a well-defined normal cone at each of its boundary points $p$. One definition of this tangent cone is the set of linear functionals on the vector space such that their restrictions to the body attain a maximum at the point $p$. The following is an adaptation of this definition to manifolds which allows us to define the tangent cones $\operatorname{Nor}_{p}\left(J^{+}(\mathcal{H})\right)$ to $J^{+}(\mathcal{H})$ at points $p \in \mathcal{H}=\partial J^{+}(\mathcal{H})$ in an invariant manner:

$$
\operatorname{Nor}_{p}\left(J^{+}(\mathcal{H})\right):=\left\{\mathrm{d} f(p): f \in C^{\infty}(M, \mathbb{R}) \text { and }\left.f\right|_{J^{+}(\mathcal{H})} \text { has a local maximum at } p\right\} .
$$

The following is a special case of a main result (see footnote 2) in the paper of Alberti [1, Theorem 3, p. 18] adapted to our notation.

Proposition 6. Let $B$ be convex body in an $(n+1)$-dimensional vector space. For each $k=1,2, \ldots, n+1$, let $\partial B[k]$ be the set of points $p \in \partial B$, so that $\operatorname{dim} \operatorname{Nor}_{p}(B) \geq k$. Then $\partial B[k]$ is a $C^{2}$ rectifiable set of dimension $n+1-k$. Therefore the Hausdorff dimension of $\partial B[k]$ is less than or equal to $n+1-k$.

Remark 7. The top and bottom dimensional cases of this are worth remarking on. When $k=n+1$ this implies that $\partial B[n+1]$ is a $C^{2}$ rectifiable set of dimension 0 . But then [1, Theorem 3 and Definition 1.1], $\partial B[n+1]$ is a countable union of sets of finite $\mathfrak{h}^{0}$ measure. However, the zero-dimensional measure $\mathfrak{h}^{0}$ is just the counting measure [9, p. 171], so that $\mathfrak{h}^{0}(A)$ is just the number of points in $A$. Therefore $\partial B[n+1]$ is countable as it is a countable union of finite sets. Consider, next, $k=1$; as $\operatorname{dim} \operatorname{Nor}_{p}\left(J^{+}(\mathcal{H})\right) \geq 1$ for all $p \in \partial B$, we have $\partial B=\partial B[1]$ and therefore $\partial B$ is a $C^{2}$ rectifiable set of dimension $n$. Because $\partial B$ is also locally the graph of a Lipschitz function, it has the further property that $\mathfrak{h}^{n}(K \cap \partial B)<\infty$ 
for all compact sets $K$. The corresponding fact is not true for $\partial B[k]$ when $2 \leq k \leq n-1$ (cf. [1, Theorem 2, p. 18]).

Theorem 3 follows immediately from this, Proposition 5, and the following lemma.

Lemma 8. For each $p \in \mathcal{H}$ the normal cone $\operatorname{Nor}_{p}\left(J^{+}(\mathcal{H})\right)$ is given by

$$
\operatorname{Nor}_{p}\left(J^{+}(\mathcal{H})\right)=\left\{\langle v, \cdot\rangle: v \in \mathcal{C}_{p}\right\} .
$$

Proof. We choose a coordinate system $x^{1}, \ldots, x^{n+1}$ on an open set $U$ containing $p$ as in Proposition 5 so that $\mathcal{H} \cap U$ is given by $x^{n+1}=h\left(x^{1}, \ldots, x^{n}\right)$, where $h$ is convex. We may assume that the point $p$ has coordinates $(0, \ldots, 0)$. We also assume that $U$ is of the form $V \times(a, b)$ for $V$ an open convex set in $\mathbb{R}^{n}$ and that $h$ takes values in the interval $(a, b)$. Then $h$ is locally Lipschitz and thus the Clarke differential $\partial h(0)$ exists and is a compact convex set of linear functionals on $\mathbb{R}^{n}[7, \mathrm{pp} .27-28]$. As $h$ is convex $\partial h(0)$ is just the set of sub-differentials to $h$ at 0 in the sense of convex analysis [7, Proposition 2.2.7, p. 36]. It follows that for $q \in \mathcal{H} \cap U$ if we write $q=(x, h(x))$ with $x \in V$ that

$$
\operatorname{Nor}_{q}\left(J^{+}(\mathcal{H})\right)=\left\{\lambda\left(\alpha-\mathrm{d} x^{n+1}\right): \lambda \geq 0, \alpha \in \partial h(x)\right\} .
$$

There is another useful description of $\partial h(0)$. Let $\Omega_{h}$ be the set of points $x$ in $V$, where the classical derivative $\mathrm{d} h(x)$ exists. As $h$ is locally Lipschitz $\Omega_{h}$ has full measure in $V$. Let $\mathcal{L}_{0}$ be the set

$$
\mathcal{L}_{0}:=\left\{\lim _{\ell \rightarrow \infty} \mathrm{d} h\left(x_{\ell}\right): x_{\ell} \in \Omega_{h}, x_{\ell} \rightarrow 0, \text { and } \lim _{\ell \rightarrow \infty} \mathrm{d} h\left(x_{\ell}\right) \text { exists }\right\},
$$

of limit points of sequences $\left\{\mathrm{d} h\left(x_{\ell}\right)\right\}$ of sequences $\left\{x_{\ell}\right\} \subset \Omega_{h}$ with $x_{\ell} \rightarrow 0$. Then [7, Theorem 2.5.1, p. 63]

$$
\partial h(0)=\text { convex hull of } \mathcal{L}_{0} .
$$

Letting, as in Section 1, $\mathcal{H}_{\text {diff }}$ be the set of points where $\mathcal{H}$ is differentiable, if $x \in V$ and $q=(x, h(x)) \in \mathcal{H}$, then $x \in \Omega_{h}$ if and only if $q \in \mathcal{H}_{\text {diff. }}$. By the theorem of Beem and Królak [2] this is the case if and only if $q$ is on exactly one generator of $\mathcal{H}$. If $q=(x, h(x)) \in \mathcal{H}_{\text {diff }}$ then let $v_{q} \in \mathcal{N}_{q}^{+}$be the unique semi-tangent to $\mathcal{H}$ at $q$. Then at $q$ the tangent plane to $\mathcal{H}$ can be defined either in terms of $\mathrm{d} h$ or in terms of $v_{q}$ to be the set of vectors $X \in T_{q}(M)$ so that $\left(\mathrm{d} h-\mathrm{d} x^{n+1}\right)(q)(X)=0$ or $\left\langle v_{q}, X\right\rangle=0$. Thus there is a positive scalar $\lambda$, so that $\left(\mathrm{d} h-\mathrm{d} x^{n+1}\right)(q)=\lambda\left\langle v_{q}, \cdot\right\rangle$. Therefore the normal cone at $q$ is one-dimensional and

$$
\operatorname{Nor}_{q}\left(J^{+}(\mathcal{H})\right)=\left\{\lambda\left\langle v_{q}, \cdot\right\rangle: \lambda \geq 0\right\}=\left\{\lambda\left(\mathrm{d} h-\mathrm{d} x^{n+1}\right): \lambda \geq 0\right\} .
$$

It follows from this that if $\left\{x_{\ell}\right\} \subset \Omega_{h}$ and $q_{\ell}=\left(x_{\ell}, h\left(x_{\ell}\right)\right)$ then $x_{\ell} \rightarrow 0$ if and only if $q_{\ell} \rightarrow p$ and $\mathrm{d} h\left(x_{\ell}\right) \rightarrow \alpha$ if and only if $v_{\ell} \rightarrow v$, where $\langle v, \cdot\rangle=\alpha$. Unraveling all this and using (3.1) and (3.2) gives that in order to complete the proof it is enough to show

$$
\mathcal{N}_{p}^{+}=\left\{\lim _{\ell \rightarrow \infty} v_{q_{\ell}}: q_{\ell} \in \mathcal{H}_{\mathrm{diff}}, q_{\ell} \rightarrow p, \text { and } \lim _{\ell \rightarrow \infty} v_{q_{\ell}} \text { exists }\right\} .
$$


Denote the right side of this equation by $\mathcal{T}_{p}$. Then [4, Lemma 6.4], the set of semi-tangents $\mathcal{N}^{+}$is a closed subset of $T(M)$ and therefore $\mathcal{T}_{p} \subseteq \mathcal{N}_{p}^{+}$. If $v \in \mathcal{N}_{p}^{+}$then there is a generator $c:[0, \infty) \rightarrow M$ with $c(0)=p, c^{\prime}(0)=v$ and parameterized so that it is unit speed with respect to the auxiliary Riemannian metric $\sigma$. For each positive integer $\ell, c(1 / \ell)$ is an interior point of the generator $c$ and thus $c(1 / \ell) \in \mathcal{H}_{\text {diff }}$. Then $v_{c(1 / \ell)}=c^{\prime}(1 / \ell)$ and $\lim _{\ell \rightarrow \infty} v_{c(1 / \ell)}=\lim _{\ell \rightarrow \infty} c^{\prime}(1 / \ell)=c^{\prime}(0)=v$. Thus $v \in \mathcal{T}_{p}$ which yields $\mathcal{N}_{p}^{+} \subseteq \mathcal{T}_{p}$. This shows (3.3) holds and completes the proof of the lemma and therefore of Theorem 3.

\section{Application to distance functions and cut loci in Riemannian manifolds}

Let $(S, h)$ be a connected Riemannian manifold which we do not assume to be complete. Let $C \subset S$ be a closed set. Then define the distance function $\rho_{C}: S \rightarrow[0, \infty)$ by

$$
\rho_{C}(p):=\text { infimum of lengths of smooth curves in } S \text { connecting } p \text { to } C \text {. }
$$

This will be Lipschitz with Lipschitz constant one: $\left|\rho_{C}(p)-\rho_{C}(q)\right| \leq d(p, q)$, where $d(p, q)$ is the Riemannian distance between $p$ and $q$.

We will see that regularity properties of $\rho_{C}$ and the cut locus of $C$ in $S$ are closely related to the regularity properties of horizons, by looking at the graph of $\rho_{C}$ (cf. Proposition 10). In this setting it is natural to consider the problem even when $(S, h)$ is not complete. For example, when $S$ is the interior of a manifold $P$ with boundary then $\rho_{C}$ agrees with the distance from $C$ defined by the infimum of the length of curves from $p$ to $C$ in $P$ so that the results apply to that case as well. Also in the setting of Lorentzian geometry one can use the graphs of functions $\rho_{C}$ to construct examples of horizons regardless of completeness of $(S, h)$.

Let $I \subset \mathbb{R}$ be an interval (which may be open, closed, or half open). Then a $C$-minimizing segment on $I$ is a unit speed geodesic $\gamma: I \rightarrow S$, so that

$$
\rho_{C}(\gamma(s))=s \quad \text { for all } s \in I .
$$

We emphasize that we do not assume that $I$ contains 0 . The Riemannian equivalent of the fact that horizons are null-geodesically ruled is contained in the following proposition.

Proposition 9. Every $p \in S \backslash C$ is on at least one $C$-minimizing segment.

Proof. Let $U$ be a convex normal neighborhood of $p$ disjoint from $C$. For $r>0$ sufficiently small the distance sphere $S_{r}(p)=\{x \in M: d(p, x)=r\}$ is contained in $U$, is compact, and agrees with the geodesic sphere of radius $r$ centered at $p$. Then, $\rho_{C}$ restricted to $S_{r}(p)$ achieves a minimum at some point $q$, say. Let $\gamma$ be the unique minimizing geodesic from $q$ to $p$. From the choice of $q$ on $S_{r}(p)$, and simple distance function considerations, one has for each $x$ on $\gamma$,

$$
d(C, q)+d(q, x)=d(C, x),
$$

where $d(C, x)=\rho_{C}(x)$. Since $\gamma$ is minimizing on each segment, Eq. (4.1) implies that $\gamma$, when suitably parameterized, is a $C$-minimizing segment. 
Each $C$-minimizing segment $\gamma: I \rightarrow S$ is contained in a maximal (with respect to the size of the interval of definition) $C$-minimizing segment and from now on we assume that all $C$-minimizing segments are defined on their maximal domain. We say that a $C$-minimizing segment $\gamma: I \rightarrow S$ has a cut point iff its interval of definition is of the form $[a, b]$ or $(a, b]$ with $b<\infty$, in which case $p=\gamma(b)$ is defined to be the cut point. A $C$-minimizing segment can fail to have a cut point either because its domain is unbounded, i.e. of the form $[a, \infty)$ or $(a, \infty)$, or because the domain is bounded, say $(a, b)$ but the $\operatorname{limit} \lim _{t \uparrow b} \gamma(t)$ does not exist in $S$. The later condition cannot arise when $S$ is complete. When $S$ is complete the domains of $C$-minimizing segments are all of the form $[0, b]$ or $[0, \infty)$.

The collection of all cut points is the cut locus of $C$ in $S$ and denoted by $\mathrm{Cut}_{C}$. The cut locus $\mathrm{Cut}_{C}$ is a subset of $S \backslash C$, and the definition here of $\mathrm{Cut}_{C}$ agrees with the usual definition when $S$ is complete. For any $p \in S \backslash C$, let $N_{C}(p)$ be the number of $C$-minimizing segments on which $p$ lies. Then for all $p \in S \backslash C$, we have $N_{C}(p) \geq 1$ and it is known that if $N_{C}(p) \geq 2$ then $p \in \mathrm{Cut}_{C}$. For $p \in S \backslash C$, let

$$
\mathcal{M}_{p}:=\left\{-\gamma^{\prime}\left(\rho_{C}(p)\right): \gamma \text { is a } C \text {-minimizing segment and } \gamma\left(\rho_{C}(p)\right)=p\right\}
$$

be the set of the unit vectors at $p$ which are tangent to $C$-minimizing segments and which point toward $C$. Then the number of vectors in $\mathcal{M}_{p}$ is just $N_{C}(p)$.

Let $M=S \times(-\infty, 0)$ and given $M$ the Lorentzian metric $g=h-\mathrm{d} t^{2}$. Let $\mathcal{H}$ be the graph of $-\rho_{C}$ in $M$, that is

$$
\mathcal{H}:=\left\{\left(x,-\rho_{C}(x)\right): x \in S \backslash C\right\} .
$$

We leave the proof of the following to the reader.

Proposition 10. The set $\mathcal{H}$ is a future horizon in $M$. The null generators of $\mathcal{H}$ are (up to reparameterization) the curves $s \mapsto\left(\gamma(s),-\rho_{C}(\gamma(s))\right)$, where $\gamma$ is $C$-minimizing segment of $S$ and $N\left(p,-\rho_{C}(p)\right)=N_{C}(p)$. The set $\mathcal{H}_{\mathrm{end}}$ coincides with the set $\left\{\left(p,-\rho_{C}(p)\right): p \in\right.$ $\left.\mathrm{Cut}_{C}\right\}$.

The theorem of Beem and Królak [2] that a point $p$ of a horizon is differentiable if and only if $N(p)=1$ implies the following Riemannian result.

Proposition 11. With notation as above, the point $p \in S \backslash C$ is a differentiable point of the distance function $\rho_{C}$ if and only if $N_{C}(p)=1$.

Remark 12. While to best of our knowledge this result has not appeared in the literature on the regularity of Riemannian distance functions, it would be surprising if it were not known, at least in the case of complete manifolds, to experts. It can also be deduced from general facts about the Clarke differential in non-smooth analysis. Explicitly, it follows from Clarke [6, Theorem 2.1(4), p. 251], a result about the generalized gradients of functions that are pointwise minimums of families of smooth functions with appropriate Hessian bounds, that the Clarke differential of $\rho_{C}$ at $x \in N \backslash C$ is

$$
\partial \rho_{C}(x)=\text { convex hull of }\left\{-\langle u, \cdot\rangle: u \in \mathcal{M}_{x}\right\}
$$


(When $S$ is Euclidean space this is [10, Lemma 4.2, pp. 1037-1038]. The extension to complete Riemannian manifolds is not hard.) The function $\rho_{C}$ is semi-convex and a semi-convex function is differentiable at $x$ if and only its Clarke differential at $x$ is a singleton. ${ }^{8}$ Therefore $\rho_{C}$ is differentiable at $x$ if and only if $N_{C}(x)=1$. It is also possible to carry out a proof of the Beem and Królak result along these lines.

In [5] horizons in Lorentzian manifolds are constructed that are non-differentiable on a dense set. Another family of such examples, possessing genericity properties, is given in [3]. When translated into Riemannian terms the examples of Chruściel and Galloway [5] imply the following proposition.

Proposition 13. There exists a closed Lipschitz curve $C \subset \mathbb{R}^{2}$ such that its distance function $\rho_{C}$ is non-differentiable on a dense subset of the set $\rho_{C} \leq 1$ of the unbounded component of $\mathbb{R}^{2} \backslash C$.

In both $[3,5]$ it is shown how to obtain higher dimensional examples. Theorem 1 implies the following proposition.

Proposition 14. If $C$ is a closed set in an n-dimensional Riemannian manifold $(S, h)$, then $\mathfrak{h}_{H}^{n}\left(\mathrm{Cut}_{C}\right)=0$.

When $C$ is a smooth submanifold of $S$ (for example when $C$ is a point) and the manifold $(S, h)$ is complete then a recent result of Itoh and Tanaka [14, Theorem B, p. 22] implies that $\mathrm{Cut}_{C}$ has Hausdorff dimension at most $n-1$. (When $S$ is two-dimensional and $C$ is a point this had been done earlier by Hebda [11] and Itoh [13].) However, for arbitrary closed sets the question of the Hausdorff dimension of $\mathrm{Cut}_{C}$ is open. In particular, it is not known if there is a closed subset $C$ of the Euclidean plane $\mathbb{R}^{2}$, with its usual metric, so that the Hausdorff dimension of $\mathrm{Cut}_{C}$ is 2. (Proposition 14 implies $\mathfrak{h}^{2}\left(\mathrm{Cut}_{C}\right)=0$, but this does not rule out the possibility that the Hausdorff dimension is 2.)

If $M=S \times(-\infty, 0)$ is given as its auxiliary Riemannian metric $\sigma=h+\mathrm{d} t^{2}$, then the set of semi-tangents to $\mathcal{H}$ at $p=\left(x,-\rho_{C}(x)\right)$ is $\mathcal{N}_{p}^{+}=\left\{\left(2^{-1 / 2} u, 2^{-1 / 2} \partial / \partial t\right): u \in \mathcal{M}_{x}\right\}$. Set

$$
\operatorname{Cut}_{C}[k]:=\left\{x \in S \backslash C: \operatorname{dim} \operatorname{Span}\left\{(u, \partial / \partial t): u \in \mathcal{M}_{x}\right\} \geq k\right\} .
$$

As in Section $1\left\{x \in S \backslash C: N_{C}(p) \geq k\right\} \subseteq \operatorname{Cut}_{C}[k]$ and equality holds if $1 \leq k \leq 3$. Note for $k \geq 2$ that $\mathrm{Cut}_{C}[k] \subset \mathrm{Cut}_{C}$, while for $k=1$, we have $\mathrm{Cut}_{C}[1]=S \backslash C$. The set $\left\{x \in N \backslash C: N_{C}(x) \geq 2\right\}=\mathrm{Cut}_{C}[2]$ is the strict cut locus and for some special choices of $C$, for example a point or a submanifold, its structure has been studied by several authors (cf. $[11,14]$ and the references therein). Using that the epigraph of a distance function has locally positive reach, it follows from results of Federer [8, Remark 4.15(3), p. 447] that each set $\mathrm{Cut}_{C}[k]$ is countable rectifiable of dimension $n+1-k$. Theorem 3 allows us to refine this to $C^{2}$ rectifiablity.

\footnotetext{
${ }^{8}$ The general semi-convex case reduces to the case of convex functions. For a convex function the Clarke differential is the sub-differential in the sense of convex analysis [7, Proposition 2.2.7, p. 36] and a convex function is differentiable at the point if and only if its sub-differential is a singleton.
} 
Proposition 15. For $1 \leq k \leq n+1$, the set $\mathrm{Cut}_{C}[k]$ is a $C^{2}$ rectifiable set of dimension $n+1-k$. Therefore $\mathrm{Cut}_{C}[k]$ has Hausdorff dimension $\leq n+1-k$.

Remark 16. By a theorem of Rademacher the set of non-differentiable points of a Lipschitz function on an $n$-dimensional manifold has $\mathfrak{h}^{n}$ measure zero. In the case that the function is a distance function, $\rho_{C}$, this can be improved. By Proposition 11 the set $\mathrm{Cut}_{C}$ [2] is exactly the set of points $p \in S \backslash C$, where the distance function $\rho_{C}$ is not differentiable. Thus, by Proposition 15, the set of non-differentiable points of $\rho_{C}$ is a $C^{2}$ rectifiable set of dimension $n-1$ and therefore has Hausdorff dimension at most $n-1$.

For each $r>0$, let $\tau_{r}(C):=\left\{p \in S: \rho_{C}(p)=r\right\}$ be the tube of radius $r$ about $C$. In general, these tubes can have singularities and need not be topological hypersurfaces in $S$. We now look at what the Riemannian versions of Chruściel et al. [4, Section 5] have to say about the regularity of $\tau_{r}(C)$ and $\rho_{C}$. By Proposition 5 the function $\rho_{C}$ is semi-convex on $S \backslash C$. Therefore by Alexandrov's theorem for almost all $p \in S \backslash C$ the function $\rho_{C}$ has second Alexandrov derivatives. This means that in local coordinates $x^{1}, \ldots, x^{n}$ centered at $p$ the function $\rho_{C}$ has a second order Taylor expansion

$$
\rho_{C}=\rho_{C}(p)+\mathrm{d} \rho_{C}(p) x+\frac{1}{2} \mathrm{D}^{2} \rho_{C}(p)(x, x)+\mathrm{o}\left(|x|^{2}\right),
$$

where $x=\left(x^{1}, \ldots, x^{n}\right), \mathrm{d} \rho_{C}(p)$ is a covector at $p$ and $\mathrm{D}^{2} \rho_{C}(p): T_{p}(S) \times T_{p}(S) \rightarrow \mathbb{R}$ is a symmetric bilinear form. Denote by $S_{\mathrm{Al}}$ the set of Alexandrov points of $\rho_{C}$. At points $p \in S_{\mathrm{Al}}$ the function $\rho_{C}$ is differentiable and therefore the discussion above yields that $N_{C}(p)=1$ and that $\mathrm{d} \rho_{C}(p)=-\langle u, \cdot\rangle$, where $u \in \mathcal{M}_{p}$. If $p \in S_{\mathrm{Al}}$ and $r=\rho_{C}(p)$ then the level set $\tau_{r}(C)$ has a well-defined tangent space $T_{p}\left(\tau_{r}(C)\right):=\left\{X \in T_{p} S: \mathrm{d} \rho_{C}(p) X=0\right\}$ and a well-defined second fundamental form $\mathbb{I}$ at $p$ given by

$$
\mathbb{I}_{p}(X, Y)=-\mathrm{D}^{2} \rho_{C}(p)(X, Y) \text { for } X, Y \in T_{p}\left(\tau_{r}(C)\right) .
$$

Let $A_{p}: T_{p}\left(\tau_{r}(C)\right) \rightarrow T_{p}\left(\tau_{r}(C)\right)$ be the corresponding Weingarten map defined by

$$
\left\langle A_{p} X, Y\right\rangle:=\mathbb{I}_{p}(X, Y) \text { for } X, Y \in T_{p}\left(\tau_{r}(C)\right) .
$$

That is $A_{p}$ is the tensor of type $(1,1)$ corresponding to the tensor $\mathbb{I}$ of type $(0,2)$ and $A_{p}$ is a self-adjoint linear map. With the choice of signs here, when $S=\mathbb{R}^{n}$ and $C$ is the origin (so that $\tau_{r}(C)$ is the sphere of radius $r$ ) for $p \in \tau_{r}(C)$, we have $A_{p}=-(1 / r) I$. Let the Riemannian connection on $S$ be denoted by $\nabla$ and let $R$ be the curvature tensor of $S$ with signs chosen so that $R(X, Y) Z=\left(\nabla_{X} \nabla_{Y}-\nabla_{Y} \nabla_{X}-\nabla_{[X, Y]}\right) Z$. For $U \in T_{p} M$ define a linear map $R_{U}: T_{p} S \rightarrow T_{p} S$ be $R_{U} X=R(X, U) U$. The following summarizes the Riemannian versions of Propositions 3.1 and 3.5 and Theorems 5.1 and 5.6 of Chruściel et al. [4].

Theorem 17. Let $\gamma: I \rightarrow S$ by a $C$-minimizing segment with $\gamma(0) \in C$. If $\gamma\left(t_{0}\right) \in S_{\mathrm{Al}}$ for some $t_{0} \in I$, then $\gamma(t) \in S_{\mathrm{Al}}$ for all $t \in I_{0}:=\left\{t_{0}\right\} \cup\left(I \cap\left[t_{0}, \infty\right)\right)^{\circ}$ (where $J^{\circ}$ is the interior of $J \subset \mathbb{R})$. The Weingarten maps $A_{\gamma(t)}$ of the tubes $\tau_{\rho_{C}(\gamma(t))}(C)$ vary smoothly on the interval $I_{0}$ and satisfy the usual Riccati equation for parallel hypersurfaces:

$$
\nabla_{\mathrm{d} / \mathrm{d} t} A_{\gamma(t)}=A_{\gamma(t)}^{2}+R_{\gamma^{\prime}(t)}
$$


Moreover, for all $r \in(0, \infty)$ the tube $\tau_{r}(C)$ has locally finite $\mathfrak{h}^{n-1}$-dimensional measure and for almost all $r \in(0, \infty)$ there is a set $P \subset \tau_{r}(C)$ with $\mathfrak{h}^{n-1}(P)=0$, so that every $C$-minimizing segment $\gamma: I \rightarrow S$ which meets $\tau_{r}(C) \backslash P$ will have $\gamma(t) \in S_{\mathrm{Al}}$ for $t \in I^{\circ}$.

In the terminology of Chruściel et al. [4] "Alexandrov points propagate to the past along generators" of horizons. In the Riemannian setting Alexandrov points of a distance function $\rho_{C}$ propagate away from $C$ along $C$-minimizing segments. The last sentence of Theorem 17 implies loosely that almost every $C$-minimizing segment is an Alexandrov segment in the sense that all of its points other than its endpoints are in $S_{\mathrm{Al}}$.

\section{References}

[1] G. Alberti, On the structure of singular sets of convex functions, Calc. Var. Partial Diff. Eqs. 2 (1) (1994) 17-27.

[2] J.K. Beem, A. Królak, Cauchy horizon end points and differentiability, J. Math. Phys. 39 (11) (1998) 60016010.

[3] R.J. Budzyński, W. Kondracki, A. Królak, On the differentiability of Cauchy horizons, J. Math. Phys. 40 (10) (1999) 5138-5142.

[4] P.T. Chruściel, E. Delay, G.J. Galloway, R. Howard, Regularity of horizons and the area theorem, Ann. H. Poincaré 2 (2001) 109-178.

[5] P.T. Chruściel, G.J. Galloway, Horizons non-differentiable on a dense set, Commun. Math. Phys. 193 (2) (1998) 449-470.

[6] F.H. Clarke, Generalized gradients and applications, Trans. Am. Math. Soc. 205 (1975) 247-262.

[7] F.H. Clarke, Optimization and Nonsmooth Analysis, 2nd Edition, SIAM, Philadelphia, PA, 1990.

[8] H. Federer, Curvature measures, Trans. Am. Math. Soc. 93 (1959) 418-491.

[9] H. Federer, Geometric Measure Theory, Die Grundlehren der Mathematischen Wissenschaften, Vol. 153, Springer, New York, 1969.

[10] J.H.G. Fu, Tubular neighborhoods in Euclidean spaces, Duke Math. J. 52 (4) (1985) 1025-1046.

[11] J.J. Hebda, Metric structure of cut loci in surfaces and Ambrose's problem, J. Diff. Geom. 40 (3) (1994) 621-642.

[12] S. Husa, J. Winicour, Asymmetric merger of black holes, Phys. Rev. D (3), 60 (8) (1999) $084019,13$. gr-qc/9905039.

[13] J. Itoh, The length of a cut locus on a surface and Ambrose's problem, J. Diff. Geom. 43 (3) (1996) 642-651.

[14] J. Itoh, M. Tanaka, The Lipschitz continuity of the distance function to the cut locus, Trans. Am. Math. Soc. 353 (1) (2000) 21-40. 